\title{
Independientemente de la cantidad, el alcohol no reduce la mortalidad
}

\author{
Irrespectively of the amount, alcohol consumption does not reduce mortality
}

Stockwell T et al, J Stud Alcohol Drugs, 2016;77(2):185-198.

\section{Objetivos}

Demostrar que el efecto protector del alcohol a bajas dosis sobre la mortalidad observado en estudios previos se debe a errores metodológicos y no a un beneficio real.

\section{Diseño}

Revisión sistemática de estudios de cohorte.

\section{Fuentes de datos}

Búsqueda en MEDLINE (PubMed) y Web of Science. Además, se hizo revisión cruzada en listas de referencia de meta-análisis previos. Las búsquedas se hicieron hasta diciembre del 2014.

\section{Selección de estudios}

Criterios de inclusión: Estudios de cohortes publicados en inglés que cuantifiquen la relación entre consumo de alcohol y mortalidad por todas las causas, que incluyan población de todas las edades, géneros, razas y culturas. Criterios de exclusión: Estudios que no separen la mortalidad de la morbilidad y aquellos realizados en poblaciones definidas como previamente enfermas.

\section{Extracción y análisis de los datos}

La extracción de datos fue realizada por dos revisores independientes. Se extrajeron los resultados medidos en riesgo relativo (RR), medidas de alcohol consumidas, características del estudio (edad de los participantes, sexo, tiempo de seguimiento, etc.), presencia de sesgos de abstemios (esto sucede cuando un ex bebedor o un bebedor ocasional es incluido en el grupo control de los no bebedores) y variables controladas en los estudios individuales.

\section{Resultados principales}

De los 2.662 estudios encontrados se seleccionaron 87, los cuales incluían una población de 3.998.626 personas, y entre las cuales se registraron 367.103 muertes. Luego de ajustar por sexo, edad al ingreso, adecuado tiempo de seguimiento, sesgo de abstemios, adecuada medición de consumo, tabaquismo y raza, no se encontró beneficio estadísticamente significativo en cuanto a reducción de mortalidad en ningún nivel de consumo. Por el contrario, se encontró un riesgo aumentado significativo de mortalidad en bebedores de consumo alto, muy alto y ex bebedores (ver tabla 1)Cuando se miden los resultados en los 6 estudios de mayor calidad pueden observarse similares resultados.

Tabla 1. Riesgo relativo de morir en los diferentes grupos de bebedores comparado con los abstemios ajustado por todas las covariables identificadas

\begin{tabular}{l|c|c}
\hline Grupos de bebedores controlados contra abstemios & RR (IC 95\%) & Valor de p \\
\hline Ex bebedores & $1,38(1,24$ a 1,54) & $<0,001$ \\
\hline Ocasionales (<1,3 g/día) & $0,95(0,85$ a 1,05) & 0,28 \\
\hline Bajo volumen (1,3 a 25 g/dia) & $0,97(0,88$ a 1,07) & 0,59 \\
\hline Volumen medio (25 a 45 g/dia) & $1,07(0,97$ a 1,18) & 0,17 \\
\hline Volumen alto (45 a 65 g/dia) & $1,24(1,12$ a 1,37) & $<0,001$ \\
\hline Muy alto volumen (>65 g/día) & $1,44(1,30$ a 1,60) & $<0,001$ \\
\hline
\end{tabular}

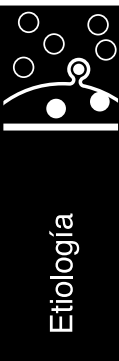

RR: riesgo relativo. IC 95\%: intervalo de confianza del $95 \%$.

\section{Conclusiones}

Luego de ajustar por las características de los estudios no se observaron beneficios estadísticamente significativos en términos de reducción de la mortalidad por todas las causas en bebedores de bajo volumen de alcohol comparado con abstemios o bebedores ocasionales.

Fuente de financiamiento de los autores: Beca aprobada por el Instituto Nacional de Salud de Estados Unidos.

\section{Comentario}

Hoy en día existe una creencia popular acerca de los beneficios cardiovasculares aportados por una medida de alcohol diaria. Esto se debe a algunos trabajos publicados en los cuales se observaba este fenómeno, amplificados por la divulgación de esta información en los medios de comunicación por parte de médicos de renombre1,2. Sin embargo la revisión sistemática resumida deja serias dudas acerca de esta asociación, ya que luego de ajustar por ciertas variables confundidoras, el beneficio del alcohol para prevenir la mortalidad por todas las causas es nulo a bajas dosis y perjudicial a dosis mayores. Esto es consecuencia principalmente de considerar a los ex bebedores dentro del grupo de abstemios, ya que, como se puede ver en el cuadro, este es el grupo de segunda mayor mortalidad dentro de los sujetos analizados, probablemente como consecuencia de haberse visto obligados a dejar la bebida por problemas de salud causados por la misma o por otras condiciones que ponen en una situación más delicada a su salud. Este último estudio tiene un mejor abordaje metodológico que sus predecesores, por lo tanto puede ser considerado la mejor evidencia al respecto hasta el momento. Sin embargo, el grado de evidencia que aporta es limitado, ya que la mayoría de los trabajos en los que se basa son estudios de cohortes, la mayoría no presenta las características adecuadas para analizar la asociación entre mortalidad y consumo de alcohol, y se observa en su conjunto un grado elevado de heterogeneidad. Esto deja la puerta abierta al debate acerca de si convendría realizar un ensayo clínico controlado y aleatorizado para evaluar el efecto del consumo de alcohol a bajas dosis, teniendo en cuenta que esto puede aportar en forma más confiable la asociación. Algunas otras limitaciones de este estudio para la aplicación de sus resultados en la práctica diaria es la falta de ajustes por tipo de bebida, dado que hay teorías sobre los beneficios de las sustancias antioxidantes del vino tinto que no pueden ser evaluadas en este análisis. Otra limitación es que la población analizada es principalmente caucásica y asiática, y no podemos decir con seguridad si los resultados son extrapolables a nuestra población. Por último se debe tener en cuenta que la asociación entre el consumo de alcohol y la morbilidad no fue medida.

\section{Conclusiones del comentador}

En base a la evidencia actual con la que contamos no estamos en condiciones de recomendar el consumo de alcohol a los pacientes, aun en bajas dosis.

Nahuel A. Orcaizaguirre. [ Servicio de Medicina Familiar y Comunitaria del Hospital Italiano de Buenos Aires. nahuel.orcaizaguirre@ hospitalitaliano. org.ar ]

Orcaizaguirre NA. Independientemente de la cantidad, el alcohol no reduce la mortalidad. Evid Act Pract Ambul. 2017;20(1):19. Comentado de: Stockwell T, y col. Do "moderate" drinkers have reduced mortality risk? A systematic review and meta-analysis of alcohol consumption and all-cause mortality. Journal of Studies on Alcohol and Drugs, 2016;77(2):185-198. PMID: 26997174. 